\title{
Reed's Syndrome: A Case of Multiple Cutaneous Leiomyomas Treated with Liquid Nitrogen Cryotherapy
}

\author{
Mohammad A. Basendwh ${ }^{a} \quad$ Mohammad Fatani $^{b} \quad$ Badee Baltow $^{c}$ \\ ${ }^{a}$ Department of Dermatology, Qunfudah Medical College, Umm Al-Qura University, \\ and Departments of ${ }^{\mathrm{b}}$ Dermatology and ${ }^{\mathrm{C}}$ Pathology, Hera General Hospital, \\ Makkah, Saudi Arabia
}

\section{Key Words}

Leiomyoma · Reed's syndrome · Cryotherapy · Painful skin lesion

\begin{abstract}
Reed's syndrome is an autosomal dominant genetic disorder. Affected individuals are at increased risk of developing benign smooth muscle tumors in the skin and uterus. In this article, we report a case of a 52-year-old female who presented to our dermatology clinic complaining of painful skin lesions on her right arm, left forearm and trunk. The patient had a past medical history of uterine leiomyomatosis for which she underwent hysterectomy 17 years ago. The patient's family history revealed that her mother, 2 sisters and 2 maternal aunts also had uterine leiomyomas. The diagnosis of Reed's syndrome was confirmed by histopathologic examination of the patient's dermal lesion in conjunction with her surgical and family histories. Five years after the initial presentation, the patient underwent treatment with liquid nitrogen cryotherapy for the dermal leiomyomas. After the treatment, marked improvement was noticed with regard to the pain and size of the skin lesions.
\end{abstract}

\section{Introduction}

The coexistence of benign smooth muscle growths in the skin and the uterus is known as Reed's syndrome. In 1973, Reed et al. [1] reported 2 families, whose successive generations demonstrated cutaneous leiomyomas, uterine leiomyomas and/or leiomyosarcomas, establishing an autosomal dominant pattern of inheritance.

\section{KARGER}


Basendwh et al.: Reed's Syndrome: A Case of Multiple Cutaneous Leiomyomas Treated with Liquid Nitrogen Cryotherapy

Cutaneous leiomyomas are benign tumors composed of smooth muscle fibers. They are classified as follows: piloleiomyomas, which originate from the erector pili; genital leiomyomas, which originate from the dartos muscle of the skin in the genital area; and angioleiomyomas, which originate from the smooth muscle of the vasculature. They belong to a painful group of skin tumors, which includes angiolipoma, dermatofibroma, eccrine spiradenoma, endometrioma, glomus tumors, granular cell tumors, neurilemmoma and neuroma [2].

Clinically, dermal leiomyomas are skin-colored or red-brown dermal papules or nodules that range from 0.2 to $2.0 \mathrm{~cm}$ in diameter, with a firm consistency. A pseudo-Darier sign, which is the transient elevation of a lesion on rubbing, may be seen in these types of lesions [3]. Leiomyomas usually remain benign. However, in the past, skin leiomyosarcomas with fumarate hydratase $(\mathrm{FH})$ germline mutation have been reported [4].

In response to pressure or cold, the skin lesions may be asymptomatic or may exhibit severe pain. However, the pathogenesis of the pain remains unknown. Some authors suggest that the pain results from local pressure or stimulation of the peripheral cutaneous nerves or by local ischemia caused by contraction of smooth muscles [5].

Here, we report a case of Reed's syndrome who was treated with cryotherapy. As the treatment progressed, a marked decline in size and severity of pain of the dermal leiomyomas was seen and reported by the patient.

The current literature pertaining to the use of liquid nitrogen cryotherapy for the treatment of multiple cutaneous leiomyomas is limited. Our review of the literature has revealed existence of only a handful of case studies, which report the effectiveness of treating dermal leiomyomas with liquid nitrogen cryotherapy. The given case study is unique as it provides an interesting clinical presentation of a disorder and a treatment method that are still not widely published.

\section{Case Presentation}

A 52-year-old Saudi woman presented to our outpatient dermatology clinic complaining of multiple painful bumps on her trunk and upper extremities, which had been present for the last 25 years. Recently, the lesions had become more painful to touch and increased in both size and number. She also had a history of uterine leiomyomatosis for which she underwent hysterectomy 17 years ago. A family history revealed that her mother, 2 sisters and 2 maternal aunts also had a history of multiple uterine leiomyomatosis.

A physical examination revealed several smooth, nonmobile nodules that were reddish to brown in color, arranged in a cluster on her right arm (fig. 1a), left forearm and trunk. The nodules were both firm and tender to touch, ranging in diameter from 0.5 to $2 \mathrm{~cm}$. The most prominent and painful nodules were removed and submitted for histological analysis, which showed a relatively well-circumscribed dermal proliferation of intersecting fascicles of spindle cells (fig. 2). The cells exhibited cigar-shaped nuclei and growth in between collagen bundles. The findings were consistent with a diagnosis of benign dermal leiomyoma.

Based on the patient's unique clinical presentation in conjunction with her surgical and family histories, a diagnosis of Reed's syndrome was confirmed. The patient was referred for genetic counseling and for evaluation of renal malignancy. Renal ultrasound and magnetic resonance imaging (MRI) were negative for renal pathology. No symptoms indicative of renal cell cancer were found.

The patient returned to the clinic after 5 years, complaining of more painful lesions.

In this instance, the patient's dermal lesions on her right arm were treated with liquid nitrogen cryotherapy. The therapy consisted of two 30-second freeze thaw cycles with a 1 
Basendwh et al.: Reed's Syndrome: A Case of Multiple Cutaneous Leiomyomas Treated with Liquid Nitrogen Cryotherapy

mm margin. After 2 weeks of cryotherapy, the patient reported a decrease in the pain and size of the lesions (fig. 1b, c). One year after cryotherapy treatment, the patient reported nonrecurrence of the lesions, with absence of pain at the site of the lesions.

\section{Discussion}

Reed's syndrome, also known as multiple cutaneous and uterine leiomyomatosis (MCUL: OMIM 150800) and hereditary leiomyomatosis and renal cell cancer (HLRCC: OMIM 605839), is a condition inherited as an autosomal dominant trait with incomplete penetrance [4].

The predisposing gene for Reed's syndrome has been localized to chromosome 1q42.343, namely, the MCUL 1 locus of the gene encoding FH. FH catalyzes the conversion of fumarate to malate in the Kreb's cycle. Along with its catalytic action, $F H$ is also believed to be a tumor suppressor gene $[2,6]$. The mutation of the $F H$ gene results in an increased risk of developing renal tumors. The most commonly associated tumor is type 2 papillary renal cell carcinoma [7].

The management of Reed's syndrome requires a multidisciplinary approach. The managing team should include a dermatologist, gynecologist, and urologic oncologist. Families may also be referred for genetic testing of germline mutations in the $F H$ gene [7]. In families with positive $F H$ gene mutation, the rate of renal cell carcinoma is about $20-25 \%$ [4]. The assessment of patients with multiple cutaneous and uterine leiomyomatosis involves a complete history and physical examination. The National Cancer Institute recommends evaluating all patients with leiomyomatosis for the presence of an occult renal malignancy [3]. However, there is no consensus regarding the screening guidelines. Renal tumors associated with Reed's syndrome can have an aggressive clinical course and may metastasize even when the tumor size is small. In MCUL patients, renal tumors can develop at an early age, and therefore, a screening plan for early detection of malignancy is proposed. The screening plan consists of renal ultrasound and MRI, starting at age 20, followed by annual renal MRI screening and semiannual renal ultrasound screening $[3,8,9]$. Computed tomography scans are not recommended for routine screening in order to reduce the chance of provoking renal cancer by inducing a second hit and decreasing the lifelong exposure to radiation $[7,8]$.

The therapy for leiomyomas includes surgical excision for symptomatic lesions, cryotherapy and carbon dioxide laser ablation, whereas medications are directed to offer pain relief for multiple painful lesions. These medications provide pain relief either by blocking smooth-muscle contractions or by targeting the activity of nerves. The medications, which block smooth muscle contractions, include nifedipine, phenoxybenzamine, nitroglycerine and doxazosin, and the medications that target the activity of the nerves include gabapentin and topical analgesics. Pharmacologic therapy has been efficacious in small studies and case reports. Recent studies also show encouraging results from using botulinum toxin injections for pain control. Botulinum toxin type A is used in different pain syndromes as it is believed to work by inhibiting the release of neuropeptides, including substance $P$ and glutamate; thus, reducing central pain signals $[3,10,11]$. Liquid nitrogen cryotherapy provides an alternative to invasive surgical techniques. Archer et al. [12] presumed that cryotherapy offers pain relief through the destruction of nerve fibers. However, this proposed mechanism needs evidence supporting the fact that cryotherapy may be directly destroying the nerve fibers, hence reducing pain rather than destroying the tumor cells/lesion Themselves/itself, hence reducing pain. 
Basendwh et al.: Reed's Syndrome: A Case of Multiple Cutaneous Leiomyomas Treated with Liquid Nitrogen Cryotherapy

\section{Conclusion}

The literature and published case studies concerning the effectiveness of liquid nitrogen cryotherapy as a treatment method for multiple symptomatic leiomyomas is scarce. In such a scenario, the given case study presents a unique opportunity to demonstrate the effectiveness of liquid nitrogen cryotherapy on dermal leiomyomas. Liquid nitrogen cryotherapy provides an alternative method for the treatment of multiple symptomatic leiomyomas. As seen in the given case study, liquid nitrogen cryotherapy is an effective noninvasive treatment modality, which can produce acceptable cosmetic results with minimal adverse effects.

\section{Statement of Ethics}

The patient gave written informed consent.

\section{Disclosure Statement}

The authors declare no conflicts of interest.

\section{References}

1 Reed WB, Walker R, Horowitz R: Cutaneous leiomyomata with uterine leiomyomata. Acta Derm Venereol 1973;53:409-416.

2 Kim G: Multiple cutaneous and uterine leiomyomatosis (Reed's syndrome). Dermatol Online J 2005;11:21.

-3 Emer JJ, Solomon S, Mercer SE: Reed's syndrome: a case of multiple cutaneous and uterine leiomyomas. J Clin Aesthet Dermatol 2011;4:37-42.

4 Makino T: Multiple cutaneous and uterine leiomyomatosis; in Oiso DN (ed): Current Genetics in Dermatology. InTech Open Access Publisher, 2013.

5 Rai VM, Balachandran C, Kudva R: Multiple painful nodules. Indian J Dermatol Venereol Leprol 2005;71:449-451.

6 Alam NA: Genetic and functional analyses of FH mutations in multiple cutaneous and uterine leiomyomatosis, hereditary leiomyomatosis and renal cancer, and fumarate hydratase deficiency. Hum Mol Genet 2003;12:1241-1252.

7 Schmidt LS, Linehan WM: Hereditary leiomyomatosis and renal cell carcinoma. Int J Nephrol Renovasc Dis 2014;7:253-260.

-8 Laufer CB, Green LB, Whittemore DE: A case of reed syndrome with a novel mutation in the fumarate hydratase gene. Case Rep Med 2013;2013:926896.

-9 Smit DL, Mensenkamp AR, Badeloe S, Breuning MH, Simon ME, van Spaendonck KY, et al: Hereditary leiomyomatosis and renal cell cancer in families referred for fumarate hydratase germline mutation analysis. Clin Genet 2011;79:49-59.

-10 Smith CG, Glaser DA, Leonardi C: Zosteriform multiple leiomyomas. J Am Acad Dermatol 1998;38(2 Pt 1):272-273.

11 Christenson LJ, Smith K, Arpey CJ: Treatment of multiple cutaneous leiomyomas with CO2 laser ablation. Dermatol Surg 2000;26:319-322.

12 Archer CB, Whittaker S, Greaves MW: Pharmacological modulation of cold-induced pain in cutaneous leiomyomata. Br J Dermatol 1988;118:255-260. 


\section{Case Reports in Dermatology}

\begin{tabular}{l|l}
\hline Case Rep Dermatol 2016;8:65-70 \\
\hline DOI: $10.1159 / 000445042$ & $\begin{array}{l}\text { C 2016 The Author(s). Published by S. Karger AG, Basel } \\
\text { www.karger.com/cde }\end{array}$ \\
\hline
\end{tabular}

Basendwh et al.: Reed's Syndrome: A Case of Multiple Cutaneous Leiomyomas Treated with Liquid Nitrogen Cryotherapy

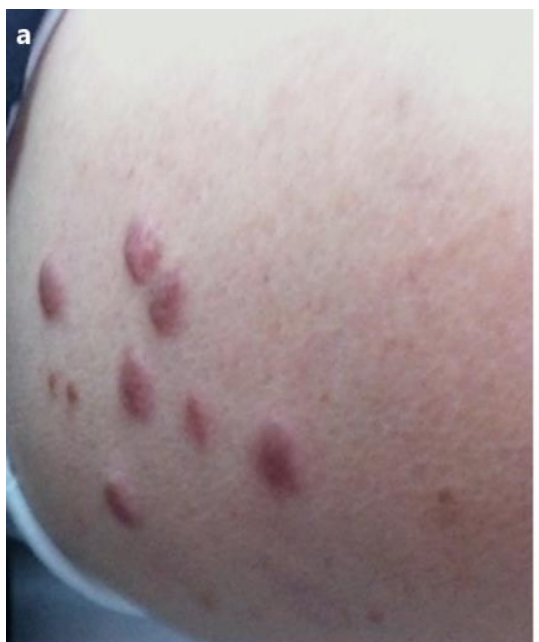

\section{b}
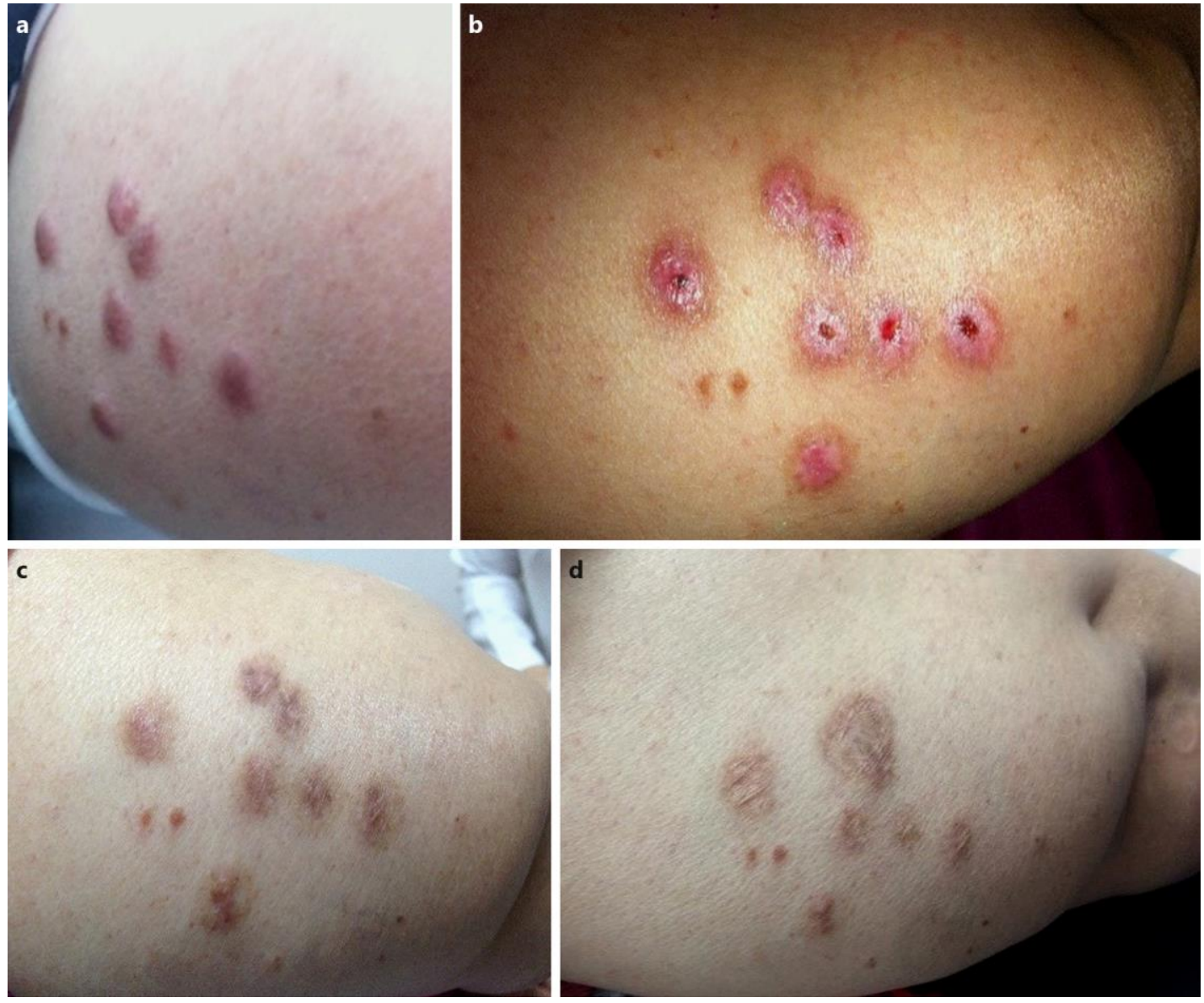

Fig. 1. a Multiple firm, flesh-colored and red-brown papules and nodules in a cluster present on the extensor surface of the patient's right arm. b Lesions after 2 weeks of liquid nitrogen cryotherapy. c Lesions after 4 weeks of liquid nitrogen cryotherapy. $\mathbf{d}$ Lesions after 1 year of liquid nitrogen cryotherapy. 
Basendwh et al.: Reed's Syndrome: A Case of Multiple Cutaneous Leiomyomas Treated with Liquid Nitrogen Cryotherapy

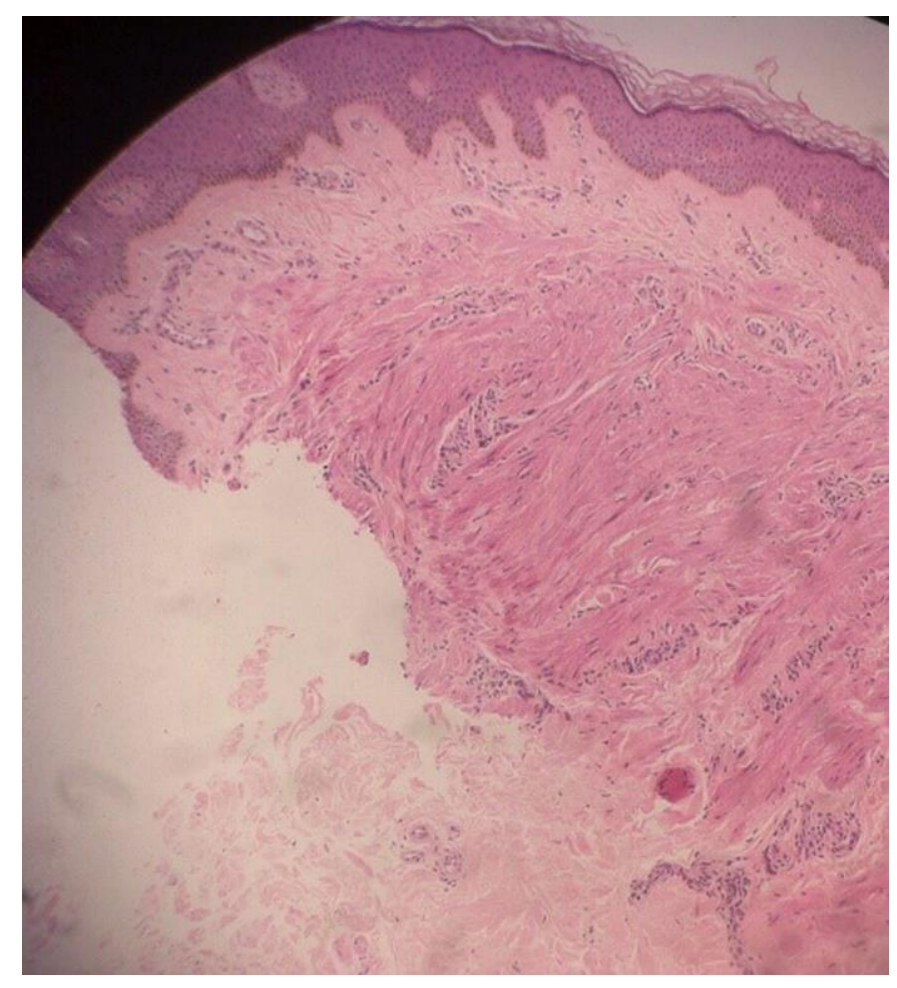

Fig. 2. There is a relatively well-circumscribed and well-defined dermal proliferation of intersecting fascicles of spindle cells. 